\title{
Predação do roedor Calomys sp. (Cricetidae) pelo marsupial Monodelphis domestica (Didelphidae) em Buíque - PE, Brasil
}

\author{
Francisco Geraldo de Carvalho Neto \\ Ednilza Maranhão dos Santos * \\ Universidade Federal Rural de Pernambuco \\ Fazenda Saco, s/n, CEP 56900-000, Serra Talhada - PE, Brasil \\ * Autor para correspondência \\ ednilzamaranhao@yahoo.com.br
}

Submetido em 02/08/2011

Aceito para publicação em 12/05/2012

\section{Resumo}

Este trabalho registra a predação de um espécime de Calomys sp. por Monodelphis domestica. O evento ocorreu no dia 30/07/2008, durante o período noturno, em área de Caatinga no Parque Nacional do Catimbau, Buíque/PE. Essa observação confirma a presença de roedores na dieta de M. domestica.

Palavras-chave: Caatinga; Catimbau; Didelphimorphia; Dieta; Mamíferos

\section{Abstract}

This paper records the predation on a specimen of Calomys sp. by Monodelphis domestica. The event took place on 07/30/2008, during the night, in a Caatinga area in Parque Nacional do Catimbau, Buique, Pernambuco, Brazil. This observation confirms the presence of rodents in the diet of $M$. domestica.

Key words: Caatinga; Catimbau; Didelphimorphia; Diet; Mammals

Monodelphis domestica (Wagner, 1842) é um marsupial, pertence à família Didelphidae, com registro para leste da Bolívia, norte do Paraguai e norte da Argentina; no Brasil ocorre nas regiões norte, sul e nordeste do Brasil (EMMONS; FEER, 1997; GARDNER, 2005; 2007; GEISE et al., 2010); é principalmente ativo durante as primeiras três horas após o anoitecer, com breves períodos de atividade durante toda a noite (STREILEIN, 1982a; 1982b; EMMONS; FEER, 1997). É uma espécie adaptada a diferentes ambientes, podendo ser encontrada em campos, cerrados, florestas úmidas e regiões semiáridas como a caatinga do nordeste brasileiro e o chaco paraguaio (GOMES, 1991).

Segundo Fonseca et al. (1996), a espécie Monodelphis domestica apresenta uma dieta insetívoraonívora; Eisenberg e Redford (1999) classificam M. domestica como um predador que se alimenta principalmente de invertebrados incluindo na sua dieta pequenos lagartos e roedores. Streilein (1982a) relata que Monodelphis domestica se alimenta de roedores vivos ou mortos, lagartos, rãs, serpentes, insetos, outros invertebrados e frutas. Já Astúa de Moraes et al. (2003) a descrevem como carnívora/insetívora. 
Calomys Waterhouse, 1837 é um gênero de pequenos roedores pertencente à família Cricetidae. Ocorre na América do Sul, principalmente em áreas secas; no Brasil são registrados ocupando áreas com vegetação aberta como Cerrado e Caatinga. Possui hábito noturno, terrestre e são principalmente granívoros (VIEIRA; BAUMGARTEN, 1995; OLIVEIRA; BONVICINO, 2006).

De uma maneira geral, informações sobre predação de mamíferos no Nordeste, principalmente para o semiárido de Pernambuco são incipientes (STREILEIN, 1982; JONES et al., 2003; VIEIRA et al., 2003). Santori e Astúa de Moraes (2006) citam a presença do gênero Calomys na alimentação de alguns Monodelphis, todavia, os autores não comentam sobre o gênero Calomys fazendo parte da dieta de $M$. domestica. Vale lembrar que para o Parque Nacional do Catimbau (PARNA Catimbau), uma área prioritária para conservação, os dados sobre a mastofauna são considerados embrionários (GEISE et al., 2010) revelando a necessidade de mais estudos.

O objetivo desse trabalho é relatar um evento de predação de um indivíduo do roedor Calomys sp. por Monodelphis domestica em área de Caatinga no Parque Nacional do Catimbau, Buíque, Pernambuco. Essa Unidade de Conservação está situada a $285 \mathrm{~km}$ de Recife, localizada numa região entre o Agreste e o Sertão de Pernambuco abrangendo os municípios de Buíque, Tupanatinga, Inajá e Ibimirim entre as coordenadas geográficas $8^{\circ} 24^{\prime} 00^{\prime \prime}$ e $8^{\circ} 36^{\prime} 35^{\prime \prime}$ 'S e $37^{\circ} 09^{\prime} 30^{\prime \prime}$ e 37¹4'40" (Figura 1A). Possui 62.300ha de área, onde se registram perto de 2.000 cavernas e 28 cavernas-cemitérios, possui uma vegetação predominantemente de caatinga arbustiva, variando entre áreas de mata primária e áreas de mata secundária, apresentando locais com resquícios de mata atlântica.O local em que o estudo foi realizado fica próximo a Pedra do Cachorro, numa área denominada "Pititi" (Figura 1B) (08 34'13,6”'S e 31 14'12,1" e elevação de 776m).

FIGURA 1: Predação de Calomys sp. por Monodelphis domestica. A= localização geográfica do PARNA Catimbau (Fonte: RODRIGUES; SANTOS, 2008); B= Paredão rochoso da localidade "Pititi"; $\mathrm{C}=$ Detalhe do ataque do marsupial à cabeça do roedor; $\mathrm{D}=$ marsupial comendo o roedor.

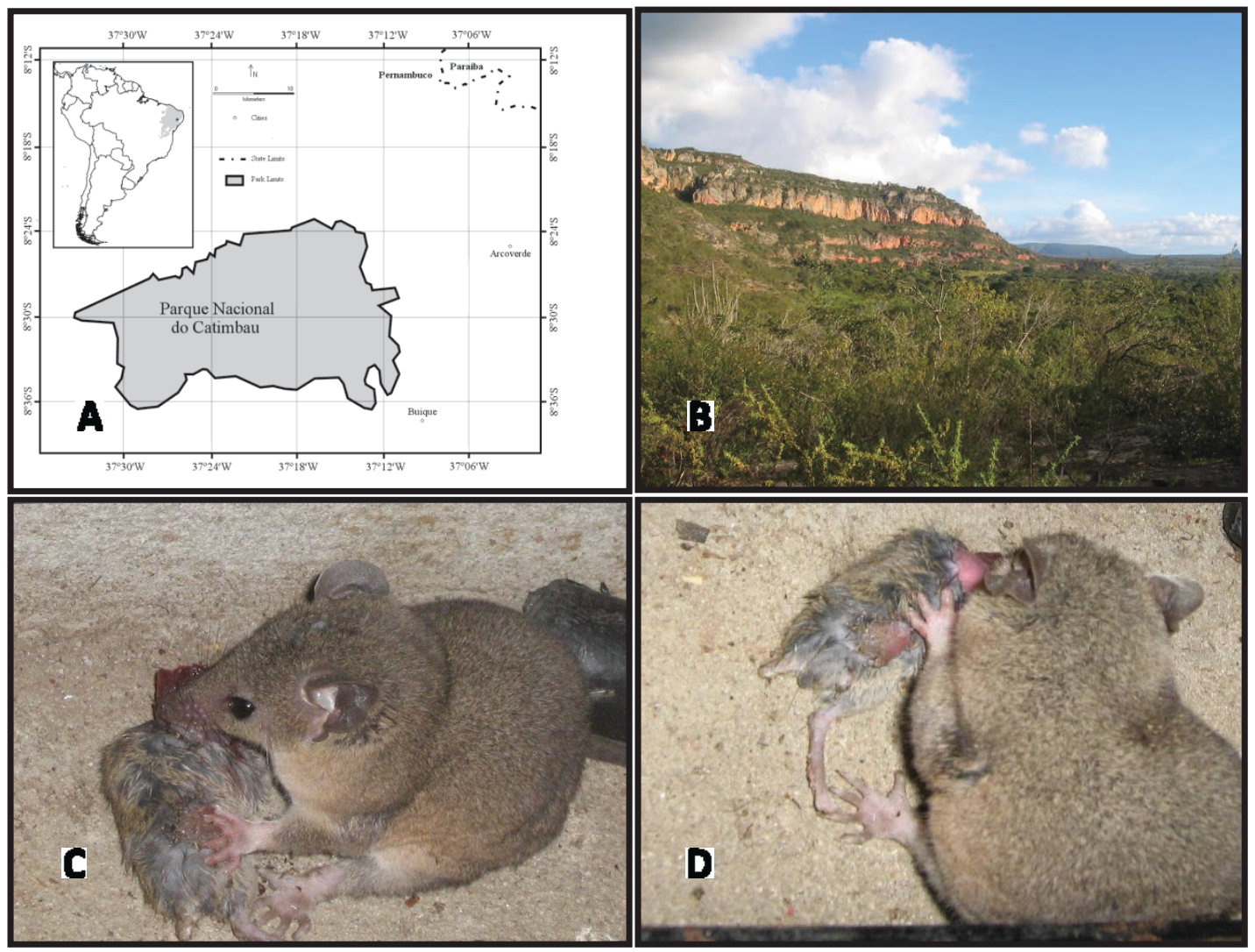


O trabalho foi realizado entre 28/07/2008 e $01 / 08 / 2008$. O evento aconteceu no dia 30 de julho de 2008 , numa noite nublada às $20 \mathrm{~h} 30 \mathrm{~min}$ com temperatura de $19,5^{\circ} \mathrm{C}$ e umidade relativa do ar de aproximadamente $85 \%$.

Através de buscas ativas, com o intuito de visualizar a fauna do PARNA Catimbau, foi possível registrar um evento de predação de um roedor Calomys sp. por um marsupial Monodelphis domestica. Foi encontrado o indivíduo de Monodelphis domestica atacando inicialmente a cabeça do roedor (Figura 1C), e depois de comê-la ingeriu o restante do corpo de forma gradativa totalizando $14 \mathrm{~min}$ de duração do evento de predação (Figura 1D).

Esse comportamento de atacar a cabeça da presa já foi observado em outros didelfídeos, como em, Monodelphis dimidiata por Gonzalez e Claramunt (2000), em Didelphis marsupialis por Wilson (1970) e em Didelphis albiventris por Oliveira e Santori (1999); provavelmente não é uma especialização para ataques a roedores, isso consiste de um padrão geral e primitivo de ataque e manipulação de presa observada em mamíferos carnívoros (OLIVEIRA; SANTORI, 1999).

O roedor possuía aproximadamente um terço do tamanho do Monodelphis domestica. Observa-se um padrão de ataque entre os didelfídeos que pode apresentar variações de postura corporal e comportamental de acordo com o tipo presa (OLIVEIRA; SANTORI, 1999). Não foi possível a identificação taxonômica a nível específico do roedor, devido o estado em que o corpo se encontrava (p.ex. apenas parte da cabeça) impossibilitando a identificação para obter medidas morfológicas do animal (Figuras 1D).

Esse registro corrobora com dados observados por Vieira e Astúa de Moraes (2003), Streilein (1982b) e Emmons e Feer (1997) que consideram Monodelphis domestica um marsupial de dieta variada, incluindo pequenos roedores e acrescenta o gênero Calomys na lista de mamíferos para o PARNA Catimbau (GEISE et al., 2010).

Atualmente, a informação existente sobre ecologia acerca da dieta de Monodelphis domestica, especificamente para os indivíduos da caatinga
(VIEIRA; ASTÚA DE MORAES, 2003; GEISE et al., 2010), são raras e incipientes destacando a relevância desse registro.

\section{Agradecimentos}

Agradecemos à equipe de Herpetologia (Sérgio Luiz da Silva Muniz, Geane Limeira da Silva, Gleymerson Vieira de Almeida e Jálber Dinarte Ramalho Magalhães) e ao Professor Dr. Diego Ástua de Moraes da Universidade Federal de Pernambuco pelas contribuições.

\section{Referências}

ASTÚA DE MORAES, D.; SANTORI, R. T.; FINOTTI, R.; CIRQUEIRA, R. Nutritional and fibre contents of laboratoryestablished diets of neotropical opossums (Didelphidae). In: JONES, M. E.; DICKMAN, C. R.; ARCHER, M. (Ed.). Predators with pouches: the biology of carnivorous marsupials. Collingwood: CSIRO Publishing, 2003. p. 221-237.

EISENBERG, J. F.; REDFORD, K. H. Mammals of the Neotropics. The Central Neotropics: Ecuador, Peru, Bolivia, Brazil. v.3. Chicago: The University of Chicago Press, 1999. p. 70-71.

EMMONS, L. H.; FEER, F. Neotropical Rainforest Mammals: A Field Guilde. 2. ed. Chicago: University of Chicago Press, 1997. $307 \mathrm{p}$.

FONSECA, G. A. B.; HERRMANN, G.; LEITE, Y. R. L.; MITTERMEIER, R. A.; RYLANDS, A. B.; PATTON; J. L. Lista anotada dos mamíferos do Brasil. Occasional Papers in Conservation Biology, Chicago, v. 4, p. 1-38, 1996.

GARDNER, A. L. Order Didelphimorphia. In: WILSON, D. E.; REEDER, D. M. (Ed.). Mammal Species of the World: a taxonomic and geographic reference. v. 1.3 ed. Baltimore: John Hopkins University Press, 2005. p. 3-18.

GARDNER, A. L. Mammals of South America. Chicago: University of Chicago Press, 2007. 669 p.

GEISE, L.; PARESQUE, R.; SEBASTIÃO, H.; SHIRAI, L. T.; ASTÚA, D.; MARROIG, G. Non-volant mammals, Parque Nacional do Catimbau, Vale do Catimbau, Buíque, state of Pernambuco, Brazil. Check List, Rio Claro, v. 6, n. 1, p. 180-186, 2010.

GOMES, N. F. Revisão sistemática do gênero Monodelphis (Didelphidae: Marsupialia). 1991. 185 f. Dissertação (Mestrado em Zoologia) - Universidade de São Paulo, São Paulo, 1991.

GONZALEZ, E. M.; CLARAMUNT, S. Behaviors of captive short-tailed opossums, Monodelphis dimidiata (Wagner, 1847) (Didelphimorphia, Didelphidae). Mammalia, Paris, v. 64, n. 3, p. 271-285, 2000.

OLIVEIRA, J. A.; BONVICINO, C. R. Ordem Rodentia. In: REIS, N. R.; PERACCHI, A. L.; PEDRO, W. A.; LIMA, I. P. (Ed.). Mamíferos do Brasil. Londrina: Editora UEL, 2006. p. 347-406. 
OLIVEIRA, M. E.; SANTORI, R. T. Predatory behavior of the opossum Didelphis albiventris (Marsupialia; Didelphidae) on the Pitviper Bothrops jararaca. Studies on Neotropical Fauna and Environment, Tübingen, v. 34, n. 2, p. 72-75, 1999.

RODRIGUES, M. T.; SANTOS, E. M. A new genus and species of eyelid-less and limb reduced gymnophthalmid lizard from northeastern Brazil (Squamata, Gymnophthalmidae). Zootaxa, Auckland, v. 1, p. 50-60, 2008.

SANTORI, R. T.; ASTÚA DE MORAES, D. Alimentação, nutrição e adaptações alimentares de marsupiais brasileiros. In: CÁCERES, N. C.; MONTEIRO FILHO, E. L. A. (Ed.). Os Marsupiais do Brasil. Campo Grande: Ed. UFMS, 2006. p. 241-254.

STREILEIN, K. E. Ecology of small mammals in the semiarid Brazilian Caatinga. I. Climate and faunal composition. Annals of Carnegie Museum, Pittsburgh, v. 51, p. 79-107, 1982a.
STREILEIN, K. E. Behavior, ecology, and distribution of South American marsupials. In: MARES, M. A.; GENOWAYS, H. H. (Ed.). Mammalian biology in South America. Vol. 6. Special publication/Pymatuning Laboratory of Ecology. Pittsburgh: University of Pittsburgh, 1982b. p. 231-250.

VIEIRA, E. M.; ASTÚA DE MORAES, D. Carnivory and insectivory in Neotropical marsupials. In: JONES, M. E.; DICKMAN, C. R.; ARCHER, M. (Ed.). Predators with pouches: the biology of carnivorous marsupials. Collingwood: CSIRO Publishing, 2003. p. 271-284.

VIEIRA, E. M.; BAUMGARTEN, L. C. Daily activity patterns of small mammals in a cerrado area from central Brazil. Journal of Tropical Ecology, Cambridge, v. 11, p. 255-262, 1995.

WILSON, D. E. Opossum predation: Didelphis on Philander. Journal of Mammalogy, Lawrence, v. 51, n. 2, p. 386-387, 1970. 